\title{
Lexical processing during saccades in text comprehension
}

\author{
KiYOMI YATABE \\ National Center of Neurology and Psychiatry, Tokyo, Japan \\ AND \\ Martin J. Pickering And Scott A. McDonald \\ University of Edinburgh, Edinburgh, Scotland
}

\begin{abstract}
We asked whether people process words during saccades when reading sentences. Irwin (1998) demonstrated that such processing occurs when words are presented in isolation. In our experiment, participants read part of a sentence ending in a high- or low-frequency target word and then made a long $\left(40^{\circ}\right)$ or short $\left(10^{\circ}\right)$ saccade to the rest of the sentence. We found a frequency effect on the target word and the first word after the saccade, but the effect was greater for short than for long saccades. Readers therefore performed more lexical processing during long saccades than during short ones. Hence, lexical processing takes place during saccades in text comprehension.
\end{abstract}

During a saccadic eye movement, visual sensitivity is greatly reduced, thereby confining the vast majority of visual perception to the fixations between saccades. This saccadic (visual) suppression means that people are unaware of the blurred motion images that would otherwise occur and are not disturbed by them. Researchers have been interested in whether higher level cognitive processing might also be suppressed during saccades. Indeed, saccades disrupt counting (Matin, Shao, \& Boff, 1993), judgments of numerical magnitude (Irwin \& Thomas, 2007) and spatial direction (Irwin \& Brockmole, 2004), mental rotation (e.g., Irwin \& Brockmole, 2000), and some shifts of spatial attention (Brockmole, Carlson, \& Irwin, 2002). However, saccades do not appear to affect other cognitive processes, such as semantic priming (e.g., Henderson, 1992) and object recognition (Irwin \& Brockmole, 2004).

To what extent do readers process text during saccades? Apart from its theoretical interest, an answer to our question is practically important, because researchers need to know whether to include the duration of saccades when measuring the reading time associated with a region of text (e.g., Rayner, 1998). This issue remains unresolved among eye movement researchers (e.g., Radach \& Kennedy, 2004; Vonk \& Cozijn, 2003).

Irwin (1998) found that the recognition and identification of isolated words occurred during saccades. He had participants fixate a box on the left of a screen. A word or pronounceable nonword appeared in this box, and at the same time, a target box appeared $7.5^{\circ}$ or $40^{\circ}$ to its right. The participants saccaded to the target box and, at the same time, pressed a button to make a lexical decision. They took equally long in both saccade condi- tions, and longer than in a control condition in which no saccade was required. Making a saccade interfered with processing, but short saccades interfered as much as long saccades. Hence, word recognition was not suppressed during the saccade (because saccade time increases in a roughly linear manner as saccade length increases). A second experiment showed similar results when the target box was presented before the word or nonword appeared. The final experiment always used a word stimulus, which the participants had to identify. At saccade onset, the word disappeared, and a visual pattern mask appeared in the target box. In most conditions, identification accuracy was greater for $40^{\circ}$ saccades than for $7.5^{\circ}$ saccades. Thus, word identification continued during the saccade.

Although this research is ingenious and convincing, it does not demonstrate what happens during saccades when people are reading sentences. Most important, the participants were reading isolated words. In addition, they were required to perform overt tasks of word recognition and identification that do not occur during normal reading. Irwin $(1998,2004)$ proposed that the duration of saccades in a region should not be subtracted from the reading comprehension time associated with that region (see also Inhoff \& Radach, 1998). But this conclusion follows only if lexical processing occurs during saccades in text comprehension.

Indeed, much evidence shows that words are processed differently in isolation from texts. First, predictions generated by the preceding context can influence the way the current word is processed and integrated with that context. Second, the perceptual span during reading includes not just the fixated word, but also the next one or two words as

K.Yatabe, yatabe@ncnp.go.jp 
well, and this parafoveal preview facilitates reading (e.g., Balota, Pollatsek, \& Rayner, 1985; see Rayner, 1998).

We therefore used sentences, as opposed to isolated words, as experimental materials. We split the sentence into two parts and required participants to make a short or a long saccade between these parts. Although extended saccades do not typically occur in most reading, such discontinuous text does occur (e.g., in advertising posters and on Web pages with many figures). In other respects, the participants read normally; in particular, they did not have to perform a secondary recognition or identification task. Our experimental logic depended on the finding that word frequency effects spill over onto the next word. Readers spend longer fixating a lower frequency word than a higher frequency one, but they also typically spend longer fixating the word following a lower frequency word than one following a higher frequency word (e.g., Henderson \& Ferreira, 1990; Kennison \& Clifton, 1995; Rayner \& Duffy, 1986).

Our intention was to use such spillover effects to investigate processing during saccades. We hypothesized that such effects would not be limited to reading continuous text but would also occur when the target word and the following word were presented at different points on the screen. If lexical processing does not occur during saccades, then the additional processing associated with low-frequency words should occur after the following word is fixated. Hence, the spillover effect should be unaffected by saccade length. But if lexical processing does occur during saccades, more of the additional processing associated with low-frequency words should take place during a longer saccade than during a shorter saccade. Thus, the spillover effect should be greater after a shorter saccade than after a longer saccade. We therefore had the participants read sentences split into two parts, with either a $10^{\circ}$ or a $40^{\circ}$ gap between them. These gaps corresponded roughly to those used by Irwin (1998), and both were much longer than the typical (forward) saccades made during reading. The participants aimed for a target box, which changed into the second part of the sentence when the participants left the target word. The final word of the first part (the target word) was either high or low frequency. We investigated whether the spillover effects on the first word of the second part (the spillover word) would be smaller for a $40^{\circ}$ gap than for a $10^{\circ}$ gap.

\section{METHOD}

\section{Participants}

Twenty-four participants (12 of them female; age, 18-33 years, $M=23.3$ ) with normal or corrected-to-normal vision were paid to take part. All were students at the University of Edinburgh whose native language was English and who had no background in psychology or linguistics.

\section{Items}

We constructed 66 pairs of items such as the following: ${ }^{1}$

1a. The large prison // remained empty.

1b. The large hangar // remained empty.

The target word was high frequency in one version (1a; prison) and low frequency in the other version (1b; hangar). The sentence was split after the target word (indicated by //).
The first part of each sentence consisted of three or four words and 15-17 characters, and the second part consisted of two to four words and 14-17 characters. Target words were 6-8 characters and two to three syllables long, with the two versions of an item being matched for characters and syllables. The mean frequency per million of the high-frequency words was 127.4 , and the mean frequency of the low-frequency words was 0.2 (Kučera \& Francis, 1967). The spillover word (e.g., remained) was 4-10 characters long. Thus, we did not expect the target or the spillover word to be skipped regularly (Rayner, 1998). In the far condition, the end of the target word was $40^{\circ}$ from the beginning of the spillover word (based on the participant's viewing distance); in the near condition, this distance was $10^{\circ}$.

Twenty-two further participants produced a word following each pretarget fragment (e.g., the large). They generated the highfrequency target word $0.4 \%$ of the time and never generated the lowfrequency target word. Another 20 participants produced a word following a fragment including the target word (with each participant encountering the version of the item with either the high-frequency target word or the low-frequency target word). The participants generated the spillover word $3.6 \%$ of the time after the high-frequency fragment (e.g., the large prison) and $3.8 \%$ of the time after the lowfrequency fragment (e.g., the large hangar). Thus, neither the target word nor the spillover word was predictable, and predictability was not affected by frequency.

We constructed four lists of experimental sentences with 16 or 17 items per condition, so that each list contained exactly one version of each item. The order of presentation was individually randomized and was preceded by four practice trials, two resembling longsaccade trials and two resembling short-saccade trials.

\section{Procedure}

The participants sat $42.5 \mathrm{~cm}$ from the display monitor with their head in a chinrest. Sentences were presented in a fixed-width font (Courier), and each character in the sentence subtended approximately $0.31^{\circ}$ of visual angle horizontally and $0.45^{\circ}$ vertically. In the far condition, the display subtended up to $50.5^{\circ}$. The stimuli were presented at a refresh rate of $120 \mathrm{~Hz}$ on a 22-in. Iiyama HM204D/ DT color monitor. Eye movements of the participants' dominant eye were recorded using an EyeLink II head-mounted eyetracker, and the eye position was sampled 500 times per second. The experimenter fully calibrated the participants' eye position at the beginning of the experiment and halfway through.

After this, a fixation square (of about a character size) appeared on the left side of the display (see Figure 1). When the participants fixated on or very close to that square for $1,500 \mathrm{msec}$, the first half of the sentence appeared so that the first letter replaced the fixation square. At the same time, a target square appeared either $10^{\circ}$ or $40^{\circ}$ away from the end of the first half of the sentence. The participants read the first half of the sentence and then fixated the target square. When the eye crossed the end of the target word, the first half of the sentence and the target square disappeared, and the second half of the sentence appeared, with the first letter of the spillover word replacing the target square. The participants then read the second half of the sentence. The participants were instructed to read as quickly but as accurately as possible and to press any key on a handheld game-pad switch as soon as they had finished reading. They then answered a simple yes/no question on a third of the trials (e.g., Was the hangar empty?) by pressing the appropriate button on the gamepad switch. The experiment lasted about $40 \mathrm{~min}$.

\section{Data Analysis}

We analyzed the target word data using first fixation time (the first fixation on the target word, including the space before it); gaze time (the sum of all fixations on the target word until the participant fixated outside the region); and total time (the sum of fixations on the target word) (see Rayner, 1998). We conducted saccade-exclusive and saccade-inclusive versions of gaze time and total time. To investigate spillover processing, we measured 
Fixation (1,500 $\mathrm{msec})$ :

\section{First Half of Sentence and Saccade Target:}

The large hangar $\quad$ OR

\section{Saccade and Second Half of Sentence:}

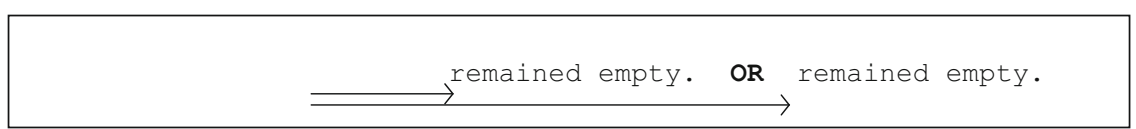

Figure 1. Schematic illustration of the experimental procedure.

elapsed time, which is the time from the beginning of the saccade launched from the target word to the beginning of the first fixation on a word after the spillover word. In other words, the elapsed time is the time of the saccade plus the saccade-inclusive gaze time on the spillover word.

We first discarded all trials on which the eye position was not recorded, trials on which the participant did not fixate the target word, and trials on which the eye regressed from the target word and launched the long saccade from a previous word $(7.6 \%$ of the trials). We then assimilated fixations that were shorter than $60 \mathrm{msec}$ and within one character space of the previous or next fixation into the largest fixation within one character space. All remaining fixations shorter than $60 \mathrm{msec}$ or longer than $1,000 \mathrm{msec}$ were removed $(0.2 \%$ of the trials). We also excluded from elapsed time $1.7 \%$ of the trials, on which the eye did not land before or on the spillover word and never came back to fixate the spillover word. In total, $9.5 \%$ of the trials were excluded from the elapsed time measure. Such trials occurred at a slightly higher rate in the high-frequency condition $(11.4 \%)$ than in the low-frequency condition $(7.8 \%)$ and in the near condition $(11.6 \%)$ than in the far condition $(7.3 \%)$.
Trials were not excluded from elapsed time measurement if the saccade from the target word resulted in a fixation on the space before the spillover word (an undershoot) or on a word following the spillover word (an overshoot) and then the spillover word was fixated afterward. These trials comprised $13.9 \%$ of the analyzed trials. Such trials occurred at a slightly higher rate in the lowfrequency condition (15.7\%) than in the high-frequency condition $(12.1 \%)$ and in the far condition $(14.0 \%)$ than in the near condition $(13.9 \%)$.

\section{RESULTS}

Table 1 reports mean times by measure and condition. For each measure, we conducted $2 \times 2$ repeated measures ANOVAs - one with participants $\left(F_{1}\right)$ and one with items $\left(F_{2}\right)$ as the random factor-with saccade target position (far vs. near) and target word frequency (high vs. low) as fixed factors. ANOVAs were carried out on the logtransformed values of the data, since D'Agostino-Pearson

Table 1

Measures of Processing Time During Reading (in Milliseconds)

As a Function of Saccade Target Position and Target Word Frequency (With Standard Errors)

\begin{tabular}{|c|c|c|c|c|c|}
\hline \multirow[b]{3}{*}{ Eyetracking Measure } & \multirow{3}{*}{$\begin{array}{c}\text { Saccade } \\
\text { Target } \\
\text { Position }\end{array}$} & \multicolumn{4}{|c|}{ Target Word Frequency } \\
\hline & & \multicolumn{2}{|c|}{ High } & \multicolumn{2}{|c|}{ Low } \\
\hline & & $M$ & $S E$ & $M$ & $S E$ \\
\hline \multirow[t]{2}{*}{ First fixation time on target word } & Far & 208 & 5 & 234 & 6 \\
\hline & Near & 213 & 5 & 232 & 5 \\
\hline \multirow[t]{2}{*}{ Gaze time on target word } & Far & 244 & 7 & 321 & 10 \\
\hline & Near & 255 & 7 & 330 & 10 \\
\hline \multirow[t]{2}{*}{ Saccade-inclusive gaze time on target word } & Far & 247 & 7 & 328 & 10 \\
\hline & Near & 259 & 8 & 337 & 11 \\
\hline \multirow[t]{2}{*}{ Total time on target word } & Far & 250 & 7 & 337 & 11 \\
\hline & Near & 265 & 8 & 356 & 14 \\
\hline \multirow[t]{2}{*}{ Saccade-inclusive total time on target word } & Far & 253 & 7 & 345 & 11 \\
\hline & Near & 269 & 8 & 364 & 15 \\
\hline \multirow[t]{2}{*}{ Elapsed time } & Far & 544 & 21 & 566 & 26 \\
\hline & Near & 398 & 17 & 474 & 25 \\
\hline \multirow[t]{2}{*}{ Gaze time on spillover word } & Far & 417 & 20 & 439 & 26 \\
\hline & Near & 344 & 17 & 418 & 24 \\
\hline \multirow[t]{2}{*}{ Saccade duration prior to spillover word } & Far & 127 & 3 & 127 & 3 \\
\hline & Near & 54 & 1 & 56 & 1 \\
\hline
\end{tabular}


tests indicated that the raw data often deviated significantly from the normal distribution and that the log-transformed values mostly did not. Accuracy on comprehension questions was uniformly high, ranging from $92.8 \%$ to $96.8 \%$ across conditions.

The participants looked longer at low-frequency target words than at high-frequency ones [first fixation time, $F_{1}(1,23)=29.11, p<.01$, and $F_{2}(1,65)=19.15, p<.01$; saccade-exclusive gaze time, $F_{1}(1,23)=76.58, p<.01$, and $F_{2}(1,65)=103.09, p<.01$; saccade-exclusive total time, $F_{1}(1,23)=79.17, p<.01$, and $F_{2}(1,65)=104.46$, $p<.01$; see Table 1]. Although the first fixation time for target words did not differ between the near and the far conditions (first fixation time: both $F \mathrm{~s}<1$ ), the gaze time and the total time were longer in the near condition than in the far condition [gaze time, $F_{1}(1,23)=4.69, p<.05$, and $F_{2}(1,65)=3.98, p<.06$; total time, $F_{1}(1,23)=8.39, p<$ .01 , and $\left.F_{2}(1,65)=6.98, p<.02\right]$. There was no interaction on any measurement (all $F \mathrm{~s}<1$ ). Thus, processing the target words displayed a standard frequency effect, and there was some indication that reading time was slightly greater before a shorter saccade than before a longer one.

Saccade-inclusive analyses of gaze time and total time produced the same results. The participants looked longer at low-frequency target words than at high-frequency ones [gaze time, $F_{1}(1,23)=78.69, p<.01$, and $F_{2}(1,65)=$ $106.51, p<.01$; total time, $F_{1}(1,23)=81.80, p<.01$, and $\left.F_{2}(1,65)=107.89, p<.01\right]$. Likewise, gaze time and total time were longer in the near condition than in the far condition [gaze time, $F_{1}(1,23)=4.55, p<.05$, and $F_{2}(1,65)=3.97, p<.06$; total time, $F_{1}(1,23)=8.18$, $p<.01$, and $\left.F_{2}(1,65)=6.99, p<.05\right]$, and there was no interaction on either measurement (all $F_{\mathrm{s}}<1$ ).

Elapsed time was longer in the low-frequency condition than in the high-frequency condition $\left[F_{1}(1,23)=13.17\right.$, $\left.p<.01 ; F_{2}(1,65)=32.90, p<.01\right]$. Thus, a spillover effect occurred after an extended saccade. Not surprisingly, elapsed time was longer in the far condition than in the near condition $\left[F_{1}(1,23)=26.49, p<.01 ; F_{2}(1,65)=\right.$ $155.95, p<.01]$. Most important, there was an interaction between target word frequency and saccade target position $\left[F_{1}(1,23)=7.30, p<.02 ; F_{2}(1,65)=7.08, p<.01\right]$. The frequency effect on spillover was $76 \mathrm{msec}$ in the near condition but only $22 \mathrm{msec}$ in the far condition.

Finally, we divided elapsed time into the (saccadeinclusive) gaze time on the spillover word and the saccade time prior to the spillover word. The gaze time was longer in the low-frequency condition than in the high-frequency condition $\left[F_{1}(1,23)=10.73, p<.01 ; F_{2}(1,65)=30.07\right.$, $p<.01]$. It was longer in the far condition than in the near condition $\left[F_{1}(1,23)=3.81, p<.07 ; F_{2}(1,65)=\right.$ $29.71, p<.01]$, although the effect was marginal by participants. Most important, it again showed an interaction between target word frequency and saccade target position $\left[F_{1}(1,23)=6.04, p<.05 ; F_{2}(1,65)=5.12, p<.05\right]$, with the frequency effect on spillover being $74 \mathrm{msec}$ in the near condition but only $22 \mathrm{msec}$ in the far condition. On the other hand, the saccade time prior to the spillover word was largely unaffected by frequency.

\section{DISCUSSION}

To determine whether readers process text during saccades, we had participants read sentences containing highor low-frequency words and then make an extended saccade to a nearer or further target location, which turned into the second part of the sentence. The participants spent longer reading the spillover word following a lowfrequency target word than following a high-frequency one. More important, they showed a larger spillover effect following a short saccade than following a long one. This result is consistent with the hypothesis that they performed more lexical processing during the long saccade than during the short one and, hence, that lexical processing is not suppressed during saccades in text comprehension.

The spillover effects seen in our experiment were relatively large, as compared with those found in previous studies (e.g., Henderson \& Ferreira, 1990; Kennison \& Clifton, 1995; Rayner \& Duffy, 1986). This was probably because the corpus frequency of the low-frequency target words used in our experiment tended to be lower than that of the target words used in the other experiments; the low-frequency target words must have required more processing time in our experiment than in the previous experiments.

The fact that gaze time on the spillover word was longer in the far than in the near condition might appear problematic for our claim that lexical processing continues during saccades. However, it is compatible with the results of studies (primarily with nonlinguistic tasks) that have demonstrated a positive correlation between saccade length and subsequent fixation duration (see Rayner, 1998).

Our findings are consistent with Irwin's (1998) conclusion that lexical processing takes place during saccades. However, they extend it in several ways. Most significantly, they demonstrate that such processing occurs during text comprehension. Although most reading clearly does not involve long saccades, our paradigm is much closer to standard reading than are paradigms involving the processing of isolated words. Moreover, we did not require participants to perform overt tasks, such as lexical decision or word identification.

We can obtain from our experimental results some insight as to the nature of spillover effects. One explanation of spillover effects is that low-frequency words require more attention than do high-frequency words and, therefore, reduce the window within which parafoveal preview benefits accrue (Henderson \& Ferreira, 1990; Kennison $\&$ Clifton, 1995). Hence, the reader is forced to do more processing on the spillover word when the target word is low versus high frequency. However, our experiment showed such a spillover effect when the spillover word was $10^{\circ}$ or $40^{\circ}$ from the target word. This is well beyond the range at which parafoveal preview operates during reading (e.g., Rayner, 1998). If our findings generalize to normal reading in this respect, they cast some doubt on this explanation of spillover effects and, instead, suggest that they occur because the eye tends to move on before all lexical processing (of low-frequency words, at least) is complete. Such results have implications for models 
of eye movement control in reading. For example, E-Z Reader (Reichle, Rayner, \& Pollatsek, 2003) models spill over as a reduction in parafoveal benefit. This mechanism cannot, therefore, explain spillover for very long saccades, as in our experiment. On the other hand, our result is compatible with the SWIFT model (Engbert, Nuthmann, Richter, \& Kliegl, 2005), in which spillover effects are claimed to be the result not only of reduced parafoveal preview, but also of time-delayed foveal inhibition, and in which it is assumed that lexical completion (as opposed to lexical preprocessing) is not paused during saccades.

In conclusion, our experimental results are consistent with the hypothesis that lexical processing takes place during saccades in text comprehension. Practically, they imply that eye movement researchers should include saccade times in measures of lexical processing within a region, because, as Vonk and Cozijn (2003) reported, the number of within-region saccades, if not the length of each such saccade, may vary according to the experimental conditions. Theoretically, they imply that the resources used for lexical processing and the resources used for driving eye movements are at least partly distinct.

\section{AUTHOR NOTE}

M.J.P. acknowledges support of a British Academy Research Readership. We thank John Henderson, Jim Brockmole, and three anonymous reviewers for comments on the manuscript. Correspondence concerning this article should be addressed to K. Yatabe, National Center of Neurology and Psychiatry, 4-1-1 Ogawa-Higashi, Kodaira, Tokyo 187-8553, Japan(e-mail: yatabe@ncnp.go.jp).

\section{REFERENCES}

Balota, D. A., Pollatsek, A., \& Rayner, K. (1985). The interaction of contextual constraints and parafoveal visual information in reading. Cognitive Psychology, 17, 364-390. doi:10.1016/0010 -0285(85)90013-1

Brockmole, J. R., Carlson, L. A., \& Irwin, D. E. (2002). Inhibition of attended processing during saccadic eye movements. Perception \& Psychophysics, 64, 867-881. doi:10.3758/PP.64.6.867

Engbert, R., Nuthmann, A., Richter, E., \& Kliegl, R. (2005). SWIFT: A dynamical model of saccade generation during reading. Psychological Review, 112, 777-813. doi:10.1037/0033-295X .112 .4 .777

Henderson, J. M. (1992). Identifying objects across saccades: Effects of extrafoveal preview and flanker object context. Journal of Experimental Psychology: Learning, Memory, \& Cognition, 18, 521-530. doi:10.1037/0278-7393.18.3.521

Henderson, J. M., \& Ferreira, F. (1990). The effects of foveal difficulty on the perceptual span in reading: Implications for attention and eye movement control. Journal of Experimental Psychology: Learning, Memory, \& Cognition, 16, 417-429. doi:10.1037/0278 $-7393.16 .3 .417$
INHOFF, A. W., \& RADACH, R. (1998). Definition and computation of oculomotor measures in the study of cognitive processes. In G. Underwood (Ed.), Eye guidance in reading and scene perception (pp. 29-54). Amsterdam: Elsevier. doi:10.1016/B978-008043361-5/50003-1

IRWIN, D. E. (1998). Lexical processing during saccadic eye movements. Cognitive Psychology, 36, 1-27. doi:10.1006/cogp.1998.0682

IRWIN, D. E. (2004). Fixation location and fixation duration as indices of cognitive processing. In J. M. Henderson \& F. Ferreira (Eds.), The interface of language, vision, and action (pp. 105-133). Hove, U.K.: Psychology Press.

Irwin, D. E., \& BRockmole, J. R. (2000). Mental rotation is suppressed during saccadic eye movements. Psychonomic Bulletin \& Review, 7, 654-661.

Irwin, D. E., \& BRockmole, J. R. (2004). Suppressing where but not what: The effect of saccades on dorsal- and ventral-stream visual processing. Psychological Science, 15, 467-473. doi:10.1111/j.0956 -7976.2004.00703.x

Irwin, D. E., \& Thomas, L. E. (2007). The effect of saccades on number processing. Perception \& Psychophysics, 69, 450-458. doi:10.3758/ PP.69.3.450

Kennison, S. M., \& Clifton, C., JR. (1995). Determinants of parafoveal preview benefit in high and low working memory capacity readers: Implications for eye movement control. Journal of Experimental Psychology: Learning, Memory, \& Cognition, 21, 68-81. doi: $10.1037 / 0278-7393.21 .1 .68$

KuČERA, H., \& FranCIS, W. N. (1967). Computational analysis of presentday American English. Providence, RI: Brown University Press.

Matin, E., Shao, K. C., \& Boff, K. R. (1993). Saccadic overhead: Information-processing time with and without saccades. Perception \& Psychophysics, 53, 372-380.

Radach, R., \& Kennedy, A. (2004). Theoretical perspectives on eye movements in reading: Past controversies, current issues, and an agenda for future research. European Journal of Cognitive Psychology, 16, 3-26. doi:10.1080/09541440340000295

RAYNER, K. (1998). Eye movements in reading and information processing: 20 years of research. Psychological Bulletin, 124, 372-422. doi:10.1037/0033-2909.124.3.372

RAYNER, K., \& Duffy, S. A. (1986). Lexical complexity and fixation times in reading: Effects of word frequency, verb complexity, and lexical ambiguity. Memory \& Cognition, 14, 191-201.

Reichle, E. D., Rayner, K., \& Pollatsek, A. (2003). The E-Z Reader model of eye movement control in reading: Comparisons to other models. Behavioral \& Brain Sciences, 26, 445-476. doi:10.1017/ S0140525X03000104

Vonk, W., \& CoziJn, R. (2003). On the treatment of saccades and regressions in eye movement measures of reading time. In J. Hyönä, R. Radach, \& H. Deubel (Eds.), The mind's eye: Cognitive and applied aspects of eye movement research (pp. 291-312). Amsterdam: Elsevier. doi:10.1016/B978-044451020-4/50017-7

\section{NOTE}

1. A complete list of items can be obtained from the corresponding author.

(Manuscript received February 8, 2008; revision accepted for publication August 12, 2008.) 